\title{
Study on Application of New Theorem of Kinetic Energy
}

\author{
Yong Yang* \\ Shanxi, 727031, PR China. \\ * Corresponding author. Tel.: +8613992969136; email: y.y.886@163.com \\ Manuscript submitted May 10, 2015; accepted March 15, 2016. \\ doi: 10.17706/ijapm.2016.6.2.31-44
}

Tongchuan Branch of Shanxi Radio and TV University, Tongchuan Polytechnics, Tongchuan New District,

\begin{abstract}
New theorem of kinetic energy (NTKE) gets rid of the imperfection that lies in the classical theorem of kinetic energy (CTKE), i.e. CTKE fails to reveal correctly that both the non-conservative force and non-conservative reacting force do dissipated work simultaneously in the equal amount, and both convert the mechanical energy of a system into non-mechanic energy of the force-bearing object. Starting with understanding the meaning of NTKE, the author makes attempts to apply NTKE on particles and on particle system to work out procedures solving physical problems, and methods to calculate terms in the new theorem and reveal the physical importance of calculated results.
\end{abstract}

Key words: New theorem of kinetic energy on particles, new theorem of kinetic energy on particle system, solving procedures, calculation of implicated work, calculation of dissipated work.

\section{Introduction}

Mechanical work is a measure of energy transferring and conversion. The basic concept in the new work-energy theory of particle dynamics includes: both conservative and non-conservative forces do non-dissipated work in such a way that the mechanical energy is transferred between particles interacting; in addition, non-conservative force does dissipated work to convert mechanical energy into non-mechanical energy of force-bearing particles; in general, non-conservative force does dissipated and non-dissipated work; non-conservative force does dissipated work and non-conservative reacting force also does dissipated work in the equal amount. The rule that force does mechanical work is expressed as new work-energy theory [1] which can objectively reveal the process of mechanical work and energy transferring and conversion as well as their relation, another upgrading of classical work-energy equation. New theorem of kinetic energy (NTKE), as a fundamental equation for new work-energy theory, must be studied and understood correctly before it is correctly applied to explain objective phenomena, provide guidance to practices and predict future.

\section{Study on Application}

\subsection{New Theorem of Kinetic Energy on Particles}

The NTKE on particles is: in the motion process of Particle $m_{\mathrm{i}}$, its increment of kinetic energy (infinitesimal) equals to the sum of total work $A_{c}$ (infinitesimal) done by conservative force, total implicated work $A_{n c i}$ (infinitesimal) done by non-conservative force, total dissipated work (infinitesimal) done by non-conservative force, and total dissipated work $A_{\text {ncrd }}$ (infinitesimal) done by non-conservative 
reacting force.

It is expressed in the equation below:

The differential formula:

$$
d A_{c}+d A_{n c i}+d A_{n c d}+d A_{n c r d}=d E_{k}
$$

The integration formula:

$$
A_{c}+A_{n c i}+A_{n c d}+A_{n c r d}=E_{k}-E_{k 0}
$$

In applying NTKE to solve practical problems, we need not only to study the forces applied to the subject but also to understand motion of each force-applying particle; in addition, we shall be able to apply correctly the new definition of mechanical work in New Work-Energy Theory to compute every term in the equation of the NTKE. Importance of NTKE is revealed in the study on applications below.

\subsection{Study on Application of NTKE on Particles}

NTKE on particles enables us to characterize transferring and conversion of mechanical energy in both moving and static particles.

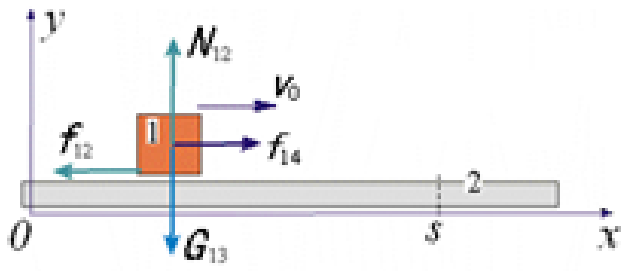

Fig. 1. Analyze forces applied to the wooden block.

Example 1: as shown in Fig. 1, a horizontal flat plate 2 in mass of $M$ is fixed on the ground; over the plate, a wooden block 1 in mass of $\mathrm{m}$ is moving towards right in straight line at a uniform speed and the coefficient of sliding friction of the wooden block 1 against the horizontal flat plate 2 is $\mu$. Question: calculate the work done by non-conservative force on Particles 1 and 2 when the wooden block 1 moves to right for the distance $s$.

Answer: using the ground as reference, establish a coordinate system 0-xy shown in Fig. 1.

Since the wooden block moves horizontally and the plate 2 is still, both can be considered as particles.

Because there is dissipating force doing work, it is solved using the NTKE on particles.

The objects interacting against each other are numbered as below: 1: the wooden block; 2: the flat plate; 3: the earth (ground); 4: the object applying pull

The meaning of the right subscript of the force $F_{i j}$ applied to particles: the first place $i$ is the number of the force-baring objects and the second place $j$ is the number of the force-applying objects $(i, j=1,2,3,4$; and $i \neq j$ ).

1) First, use the motion particle 1 as the subject to explore the relations about work-energy transferring and conversion.

- Analyze forces applied to Particle 1: The gravity $G_{13}$, the supporting force $N_{12}$ by the flat plate, the friction $f_{12}$ against the flat force and the pull $f_{14}$, the last three of which are non-conservative force.

Their respective reacting forces are, in turn: the gravity $G_{31}$ from the particle, the pressure $N_{21}$ and the friction $f_{21}$ against Particle 2 , and the pull $f_{41}$ against the force-applying object. 
- Since Particle 1 moves in a straight line at a uniform speed, the equation of Newton's Second Law for Particle 1 is:

$$
\left\{\begin{array}{c}
G_{13}+N_{12}+f_{12}+f_{14}=m a_{1} \\
a_{1}=0
\end{array}\right.
$$

The equations set consisting of respective component equations and auxiliary equations is:

$$
\left\{\begin{array}{c}
\sum_{\mathrm{j}=2}^{4} F_{x 1 \mathrm{j}}=-f_{12}+F_{14}=0 \\
\sum_{\mathrm{j}=2}^{4} F_{y 1 \mathrm{j}}=N_{12}-G_{13}=0 \\
f_{12}=f_{21}=\mu N_{12} \\
N_{21}=N_{12} \\
G_{13}=m g
\end{array}\right.
$$

Solve the above-stated equation set to obtain:

$$
\left\{\begin{array}{l}
N_{21}=N_{12}=G_{13}=m g \\
f_{14}=f_{12}=f_{21}=\mu m g
\end{array}\right.
$$

- In the process that Particle 1 moves along the positive direction of Axis $x$ in the distance $s$, the work done by forces on Particle 1 is:

The work done by non-conservative forces is to be calculated with the classical definition of the mechanical work: integrate the product of the conservative-force vector point and the infinitesimal of the absolute displacement made by the force-bearing particle [2], which is a non-dissipated work.

$$
A_{\mathrm{cl}}=A_{\mathrm{cl} 3}=\int_{r_{10}}^{r_{10}+\mathrm{s} i} G_{13} \cdot \mathrm{d} r_{1}=\int_{x_{10}}^{x_{10}+\mathrm{s}} \mathrm{G}_{13} \cos \frac{\pi}{2} \mathrm{~d} r_{1}=\int_{x_{10}}^{x_{10}+\mathrm{s}} 0 \mathrm{~d} r_{1}=0
$$

Then calculate the works of Particle 1 done by non-conservative forces, which shall be calculated in accordance with the new definition of work and include the dissipated work and the implicated work.

Calculate the implicated work by the non-conservative force: integrate the product of the vector point of the non-conservative forces and the infinitesimal of the implicated displacement of the force-bearing particle [3] (the absolute displacement of the force-applying particle), which is a non-dissipated work.

$$
\begin{gathered}
A_{n c i 12(1)}=\int_{r_{20}}^{r_{20}} N_{12} d r_{2} \\
A_{n c i 12(2)}=\int_{r_{20}}^{r_{20}} f_{12} d r_{2} \\
A_{n c i 14}=\int_{r_{40}}^{r_{40}+s i} f_{14} \cdot d r_{4}=\int_{x_{40}}^{x_{40}+s} f_{14} \cos 0 d x_{4}=f_{14} s(=\mu m g s)
\end{gathered}
$$


Substitute Equations (7)-(9) into the equation below, to obtain the total implicated work of Particle 1 done by non-conservative forces, as below:

$$
\begin{aligned}
& A_{n c i 1}=\sum_{\mathrm{j}=2}^{4} A_{n c i 1 j} \\
& =A_{n c i 12(1)}+A_{n c i 12(2)}+A_{n c i 13}+A_{n c i 14} \\
& =0+0+0+f_{14} s \\
& =f_{14} s(=\mu m g s)
\end{aligned}
$$

Calculate the dissipated work by the non-conservative force: integrate the product of the vector point of the non-conservative forces and the half of the infinitesimal of the relative displacement of the force-bearing particle relative to the force-applying particle [4], [5].

$$
\begin{gathered}
A_{n c d 12(1)}=\int_{r_{120}}^{r_{120}+s i} \frac{1}{2} N_{12} \cdot d r_{12}=\int_{x_{120}}^{x_{120}+s} \frac{1}{2} N_{12} \cos \frac{\pi}{2} d x_{12}=\int_{x_{120}}^{x_{120}+s} 0 d x_{12}=0 \\
A_{n c d 12(2)}=\int_{r_{120}}^{r_{120}+s} \frac{1}{2} f_{12} \cdot d r_{12}=\int_{x_{120}}^{x_{120}+s} \frac{1}{2} f_{12} \cos \pi d x_{12}=\frac{1}{2} \int_{x_{120}}^{x_{120}+s}(-\mu m g) d x_{12}=-\frac{1}{2} \mu m g s \\
A_{n c d 14}=\int_{r_{140}}^{r_{140}} \frac{1}{2} f_{14} \cdot d r_{14}=0
\end{gathered}
$$

There is no relative displacement of Particle 1 relative to the pull-applying point.

Substitute Equations (11)-(13) into the equation below, to obtain the total dissipated work of Particle 1 done by non-conservative forces, as below:

$$
\begin{aligned}
& A_{n c d 1}=\sum_{\mathrm{j}=2}^{4} A_{n c d 1 j} \\
& =A_{n c d 12(1)}+A_{n c d 12(2)}+A_{n c d 13}+A_{n c d 14} \\
& =0+0+0+\left(-\frac{1}{2} \mu m g s\right) \\
& =-\frac{1}{2} \mu m g s
\end{aligned}
$$

The above-stated equation represents the dissipated work of the particle 1 by non-conservative forces, and through the process the mechanical energy is converted into the thermal energy and the deformation energy in the particle 1 to which forces are applied.

Calculate the dissipated work by non-conservative reacting forces

From the definition formula of the dissipated work below [5]

$$
d A_{n c d i j}=\frac{1}{2} f_{i j} \cdot d r_{i j}=\frac{1}{2}\left(-f_{j i}\right)-d r_{j i}=\frac{1}{2} f_{j i} d r_{j i}=d A_{n c r d j i}
$$

It is known that, the dissipated work of the force-applying particle done by non-conservative reacting 
forces equals to the dissipated work of Particle 1 done by corresponding non-conservative forces, each is:

$$
\begin{gathered}
A_{n c r d 21(1)}=A_{n c r d 12(1)}=0 \\
A_{n c r d 21(2)}=A_{n c r d 12(2)}=-\frac{1}{2} \mu m g s
\end{gathered}
$$

The above-stated equation represents the dissipated work of the particle 2 by non-conservative reacting forces, and through the process the mechanical energy is converted into the thermal energy and the deformation energy in the particle 2 to which forces are applied.

$$
A_{n c r d 41}=A_{n c d 14}=0
$$

Substitute Equations (16) (18) into the equation below, to obtain the total dissipated work of force-applying particles done by non-conservative reacting forces, as below:

$$
\begin{aligned}
& A_{n c r d 1}=\sum_{\mathrm{j}=2}^{4} A_{n c r d j 1} \\
& =A_{n c r d 21(1)}+A_{n c r d 21(2)}+A_{n c r d 31}+A_{n c r d 41} \\
& =A_{n c r d 12(1)}+A_{n c r d 12(2)}+A_{n c r d 13}+A_{n c r d 14} \\
& =\sum_{j=2}^{4} A_{n c r d 1 j} \\
& =A_{n c r d 1} \\
& =-\frac{1}{2} \mu m g s
\end{aligned}
$$

That is to say, the total dissipated work $A_{n c d}$ of the particle 1 by non-conservative forces equals to the total dissipated work $A_{n c d}$ of all force-applying particles by non-conservative reacting forces.

- Calculate the increment of kinetic energy in Particle 1

Particle 1 makes linear motion at an uniform speed:

$$
E_{k 1}=E_{k 10}=\frac{1}{2} m v_{0}^{2} \rightarrow \Delta E_{k 1}=E_{k 1}-E_{k 10}=0
$$

- Use equations to solve

Substitute Equations (6), (10), (14), (19) and (20) into the Equation (2) - the NTKE for particles - to obtain:

$$
f_{14} s+\left(-\frac{1}{2} \mu m g s\right)+\left(-\frac{1}{2} \mu m g s\right)=0
$$

The right part to the equation's equal sign indicates: the increment of kinetic energy in Particle 1 is zero. The left part to the equation's equal sign indicates: total mechanical energy (value: $\mu m g s$ ) of Particle 1 
converted from the non-dissipated work of Particle 1 by pull, half of which, due to the dissipated work (the 2nd term) done by non-conservative forces, is converted into the non-mechanical energy (value: $\frac{1}{2} \mu m g s$ ) of the force-bearing particle, and another half, due to the dissipated work (the 3rd term) of the force-applying particle 2 by the non-conservative forces, is converted into the non-mechanical energy [6] (value: $\frac{1}{2} \mu m g s$ ) of the force-bearing particle 2 . Simultaneously when the mechanical energy is progressively transferred to the particle 1, it is converted into non-mechanical energy of objects with mutual friction instantly in equal amount; and the transferred mechanical energy stream and the converted non-mechanical energy stream are expressed with separate terms so as to show distinct corresponding relationship and clear implications of each term. Since it observes to the energy conversion and conservation law, the ZTKE on particle applies universally in the particle dynamics in the general condition that both the conservative force and the non-conservative force are present.

What if Particle 1 makes a linear motion at a varying speed rather than at an uniform speed?

When Particle 1 is accelerating:

$$
\Delta E_{k 1}=E_{k 1}-E_{k 10}>0
$$

Then

$$
f_{14} s+\left(-\frac{1}{2} \mu m g s\right)+\left(-\frac{1}{2} \mu m g s\right)>0
$$

It indicates that, of the total mechanical energy of Particle 1 transferred from the non-dissipated work of Particle1 (the 1st term) by the pull, some is converted into non-mechanical energy (values are $\frac{1}{2} \mu m g s$, respectively) of Particles 1 and 2 in mutual friction, and the rest is transferred to Particle 1 which is in motion resulting in increased kinetic energy in Particle 1.

When Particle 1 is decelerating:

$$
\Delta E_{k 1}=E_{k 1}-E_{k 10}<0
$$

Then

$$
f_{14} s+\left(-\frac{1}{2} \mu m g s\right)+\left(-\frac{1}{2} \mu m g s\right)<0
$$

It indicates that a pair of friction forces makes dissipated work to convert the mechanical energy into non-mechanical energy of Particles 1 and 2 in mutual friction, the value of both being $\frac{1}{2} \mu m g s$, and some of the converted mechanical energy comes from the non-dissipated work (the 1st term) of Particle 1 by the pull being transferred to Particle 1 and the rest is at the cost of decreased kinetic energy in Particle 1 in motion.

2) Then, use the static particle 2 as subject to discuss the relationship in mechanical energy 
transferring and conversion.

As shown in Fig. 2.

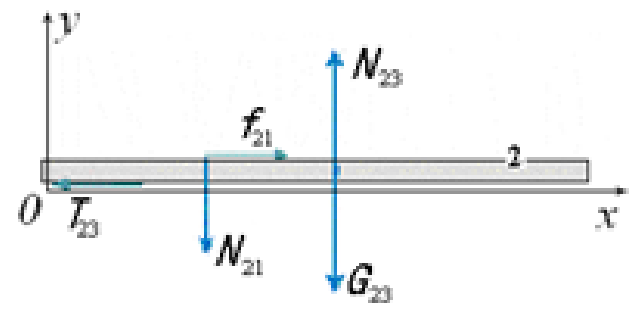

Fig. 2. Analyze forces applied to the flat plate.

- Analyze the forces applied to Particle 2: the gravity $G_{23}$, the ground supporting force $N_{23}$, the binding force $T_{23}$, the friction force $f_{21}$ against Particle 1 , and the positive pressure $N_{21}$ from Particle 1, of which the last four are non-conservative force.

- Since Particle 2 is still, the equation of Newton's Second Law on Particle 2 and the auxiliary equations are presented below:

$$
\left\{\begin{array}{c}
G_{23}+N_{23}+T_{23}+N_{21}+f_{21}=m a_{2} \\
a_{2}=v_{2}=0 \\
G_{23}=M g \\
f_{21}=f_{12}=\mu m g \\
N_{21}=N_{12}=m g
\end{array}\right.
$$

By solving the component's equation set of the above-stated vector equation set, the following is obtained:

$$
\left\{\begin{array}{c}
T_{23}=f_{21}=\mu m g \\
N_{21}=m g \\
N_{23}=(m+M) g
\end{array}\right.
$$

- In the process when the force-applying Particle 1 moves along the positive direction of Axis $x$ in the distance $s$, the work done by forces on Particle 2 is:

The work by the conservative forces

$$
A_{\mathrm{c} 2}=A_{\mathrm{c} 23}=\int_{x_{20}}^{x_{20}} G_{23} \cdot d r_{2}=0
$$

Then calculate the work of Particle 1 by non-conservative forces

Calculate the implicated work by non-conservative forces

$$
\begin{aligned}
& A_{n c i 21(1)}=\int_{r_{10}}^{r_{10}+s i} N_{21} \cdot d r_{1}=\int_{x_{10}}^{x_{10}+s} N_{21} \cos \left(-\frac{\pi}{2}\right) d x_{1}=\int_{x_{10}}^{x_{10}+s} 0 d x_{1}=0 \\
& A_{n c i 21(2)}=\int_{r_{10}}^{r_{10}+s i} f_{21} \cdot d r_{1}=\int_{x_{10}}^{x_{10}+s} f_{21} \cos 0 d x_{1}=f_{21} s(=\mu m g s)
\end{aligned}
$$




$$
A_{n c i 23(1)}=\int_{r_{30}}^{r_{30}} N_{23} \cdot d r_{3}=0
$$

The force-applying object, i.e. the ground, is the reference and therefore has no absolute displacement. Refer to 1.3.2.1.

$$
A_{n c i 23(2)}=\int_{r_{30}}^{r_{30}} T_{23} \cdot d r_{3}=0
$$

Substitute Equations (29)-(32) into the equation below, to obtain the total implicated work of Particle 2 done by non-conservative forces, as below:

$$
\begin{aligned}
& A_{n c i 2}=\sum_{j=1, \neq 2}^{3} A_{n c i 2 j} \\
& =A_{n c i 21(1)}+A_{n c i 21(2)}+A_{n c i 23(1)}+A_{n c i 23(2)} \\
& =f_{21} s(=\mu m g s)
\end{aligned}
$$

Calculate the dissipated work by non-conservative forces

$$
\begin{gathered}
A_{n c d 21(1)}=\int_{r_{210}}^{r_{210}-s i} \frac{1}{2} N_{21} \cdot d r_{21}=\int_{x_{210}}^{x_{210}-s} \frac{1}{2} N_{21} \cos \left(-\frac{\pi}{2}\right) d x_{21}=\int_{x_{210}}^{x_{210}+s} 0 d x_{21}=0 \\
A_{n c d 21(2)}=\int_{r_{210}}^{r_{210}-s i} \frac{1}{2} f_{21} \cdot d r_{21}=\int_{x_{210}}^{x_{210}-s} \frac{1}{2} f_{21} \cos 0 d x_{1}=-\frac{1}{2} \mu m g s \\
A_{n c d 23(1)}=\int_{r_{230}}^{r_{230}} \frac{1}{2} N_{23} \cdot d r_{23}=0 \\
A_{n c d 23(2)}=\int_{r_{230}}^{r_{230}} \frac{1}{2} T_{23} \cdot d r_{23}=0
\end{gathered}
$$

Substitute Equations (34)-(37) into the equation below, to obtain the total dissipated work of Particle 2 done by non-conservative forces, as below:

$$
\begin{aligned}
& A_{n c d 2}=\sum_{j=1, \neq 2}^{3} A_{n c d 2 j} \\
& =A_{n c d 21(1)}+A_{n c d 21(2)}+A_{n c d 23(1)}+A_{n c d 23(2)} \\
& =-\frac{1}{2} \mu m g s
\end{aligned}
$$

Calculate the dissipated work by non-conservative reacting forces

The following is obtained from Equation (19):

$$
A_{n c r d 2}=A_{n c d 2}=-\frac{1}{2} \mu m g s
$$


- Calculate the increment of kinetic energy in Particle 2

When Particle 1 is still:

$$
E_{k 2}=E_{k 20}=\frac{1}{2} M 0^{2}=0 \rightarrow \Delta E_{k 2}=E_{k 2}-E_{k 20}=0
$$

- Use equations to solve

Substitute Equations (28), (33), (38), (39) and (40) into the Equation (2) - the NTKE for particles - to obtain:

$$
f_{21} s+\left(-\frac{1}{2} \mu m g s\right)+\left(-\frac{1}{2} \mu m g s\right)=0
$$

The right part to the equation's equal sign indicates: in the moving process of the force-applying object, the increment of kinetic energy in Particle 2 which is still and under force is zero.

The left part to the equation's equal sign indicates: total mechanical energy (value: $f_{21} s=\mu m g s$ ) of Particle 2 converted from the non-dissipated work of Particle 2 by pull, half of which, due to the dissipated work (the 2nd term) done by non-conservative forces, is converted into the non-mechanical energy (value: $\frac{1}{2} \mu m g s$ ) of the force-bearing particle, and another half, due to the dissipated work (the 3rd term) of the force-applying particle 1 by the non-conservative forces, is converted into the non-mechanical energy [4] (value: $\frac{1}{2} \mu m g s$ ) of the force-bearing particle 1 .

Objective characterization of the transferring and conversion of the still Particle 2 that is acted by sliding friction force cannot be presented with the classical kinetic energy theorem because in terms of CTKE, the work-energy equation for the still Particle 2 is:

$$
0=0
$$

It is impossible to get data about transferring and conversion of mechanical energy in the still Particle 2 that is under the sliding friction force using the Equation (42). Therefore, wherever any non-conservative force is present, the NTKE on particle, rather than the CTKE, shall be used, because the latter is applicable in the case that there exists no conservative force.

\subsection{New Theorem of Kinetic Energy on Particle System}

The NTKE on particle system is expressed as: in the motion process of a system, the sum of works $A_{\mathrm{c}}$ (infinitesimal) of a particle system by all conservative forces, the dissipated work $A_{n c d}$ (infinitesimal) by all non-conservative forces, the dissipated work $A_{n c r d}$ (infinitesimal) by all non-conservative reacting forces, and the implicated work $A_{n c i}^{e x}$ (infinitesimal) by all non-conservative external forces equals to the increment (infinitesimal) of total kinetic energy in the system.

The differential expression is:

$$
d A_{c}+d A_{n c d}+d A_{n c r d}+d A_{n c i}^{e x}=d E_{k}
$$

The integral expression is: 


$$
A_{c}+A_{n c d}+A_{n c r d}+A_{n c i}^{e x}=E_{k}-E_{k 0}
$$

where,

$$
\begin{gathered}
A_{c}=A_{c}^{i n}+A_{c}^{e x}\left(d A_{c}=d A_{c}^{i n}+d A_{c}^{e x}\right) \\
A_{n c d}=A_{n c d}^{i n}+A_{n c d}^{e x}\left(d A_{n c d}=d A_{n c d}^{i n}+d A_{n c d}^{e x}\right) \\
A_{n c r d}=A_{n c r d}^{i n}+A_{n c r d}^{e x}\left(d A_{n c r d}=d A_{n c r d}^{i n}+d A_{n c r d}^{e x}\right)
\end{gathered}
$$

In the above-presented equations, terms with the pre-superscript "in" are works associated with the internal forces within the system while the terms with the pre-superscript "ex" are works corresponding to the external forces. There is no total implicated work by non-conservative internal forces, indicating that the implicated work associated with the non-conservative internal forces are the values of mechanical energy transferred between particles within the system, which does not change the total kinetic energy in the system.

\subsection{Exploration on Application of NTKE on Particle System}

Example 2: as shown in Fig.3, two objects $A$ and $B$ in respective mass of $m_{1}$ and $m_{2}$ are hung on both sides of the light pulley. $A$ is placed over the slope at the angle $\theta$ and the friction factor between $A$ and the slope is $\mu$. Solve the works done by all forces and the end speeds of two particles when $B$ accelerates downwards starting with stillness by height of $\Delta h$ ? (assuming the elongation of the light rope is negligible and the axis of the light pulley is smooth).

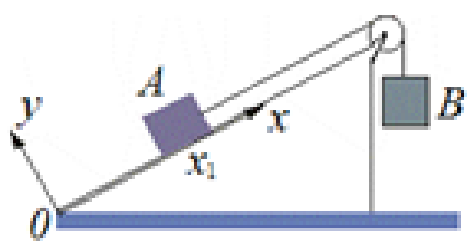

Fig. 3. Particle groud motion.

Answer: use the NTKE on particle system to solve.

Using the slope (the Earth) as the reference, set up a 0-xy coordinate system.

Because both A and B moves in linear way, both can be treated as particle.

Particles A and B constitute a system.

Define the number of objects interacting against each other: 1: Particle A; 2: Particles B; 3: the light rope; 4: the slope (the Earth).

1) Analyze the forces borne by members of the system

Forces borne by Particle $A$ at the time $t$ when it moves to the position $\left(x_{1}, 0\right)$ :

There is no internal force between Particles A and B.

System's external forces:

The pull from the light rope: $f_{13}$; the gravity $F_{14(1)}=m_{1} g$; the support force of the slope: $f_{14(2)}$ and the slope friction force: $f_{14(3)}$.

The non-conservative external reacting forces include: 
The pull of Particle A relative to the light rope: $f_{31}$; the pressure against the slope: $f_{41(2)}$; and the friction force against the slope: $f_{41(3)}$.

External forces borne by the system at the time t when Particle B moves to the position $\left(x_{2}, y_{2}\right)$ :

The gravity $F_{24}=m_{2} g$ and the pull of the light rope $f_{23}$.

The non-conservative external reacting forces include:

The pull of Particle B relative to the light rope $f_{32}$

2) Since Particles $A$ and $B$ do accelerating linear motion respectively, the component equations for Newton's Second Law and the auxiliary equation sets are presented below:

$$
\left\{\begin{array}{c}
\text { PartcileA }:\left\{\begin{array}{c}
\sum F_{x}=f_{13}-f_{14(3)}-F_{14(1)} \sin \theta=m_{1} a_{1} \\
\sum F_{\mathrm{y}}=f_{14(2)}-F_{14(1)} \cos \theta=0 \\
f_{14(3)}=f_{41(3)}=\mu f_{14(2)} \\
F_{14(1)}=m_{1} g
\end{array}\right. \\
\text { PartcileB }:\left\{\begin{array}{c}
F_{24}-f_{23}=m_{2} a_{2} \\
F_{24}=m_{2} g \\
a_{1}=a_{2} \\
f_{14(2)}=f_{41(2)} \\
f_{13}=f_{31}=f_{32}=f_{23}
\end{array}\right.
\end{array}\right.
$$

By solving the above-presented equation set, the following is obtained:

$$
\left\{\begin{array}{c}
a_{1}=a_{2}=\frac{m_{2}-m_{1}(\sin \theta+\mu \cos \theta)}{m_{1}+m_{2}} g \\
f_{13}=f_{31}=f_{23}=f_{32}=\frac{m_{1} m_{2} g}{m_{1}+m_{2}}(1+\sin \theta+\mu \cos \theta) \\
f_{14(2)}=f_{41(2)}=m_{1} g \cos \theta \\
f_{14(3)}=f_{41(3)}=\mu m_{1} g \cos \theta
\end{array}\right.
$$

3) Because both particles do linear motions respectively and all forces borne are constant, the method to calculate the work by constant-forces, can be used, in combination with the new definition equation of respective works, to calculate.

While Particle B starts from stillness and accelerates downwards by height of $\Delta \mathrm{h}$,Particle A starts from stillness to accelerate in linear way along the slope at the displacement of $\Delta x=\Delta h$.

Based on the Equation (38) for NTKE on particle system, the works are calculated:

- Total work of the system by the conservative forces:

$$
A_{c}=A_{c}^{i n}+A_{c}^{e x}
$$

The internal force is nonexistent: 


$$
\begin{gathered}
A_{c}^{i n}=0 \\
A_{c}^{e x}=A_{c 14(1)}^{e x}+A_{c 24}^{e x} \\
=-m_{1} g \sin \theta \Delta x+m_{2} g \Delta h \\
=\left(m_{2}-m_{1} \sin \theta\right) g \Delta h
\end{gathered}
$$

Substitute Equations (51) and (52) into (45), we obtains:

$$
A_{c}=\left(m_{2}-m_{1} \sin \theta\right) g \Delta h .
$$

The conservative external forces do non-dissipated work to the particle system to transfer the mechanical energy to the particle system.

- Calculate total dissipated work of the system by the conservative forces:

$$
A_{n c d}=A_{n c d}^{i n}+A_{n c d}^{e x} .
$$

The internal force is nonexistent:

$$
A_{n c d}^{\text {in }}=0
$$

Total dissipated work of the system by the non-conservative forces:

Terms with known value:

No relative displacement of the particle's force-bearing point relative to the rope's force-applying point.

$$
\begin{gathered}
\Delta x_{13}=\Delta x_{23}=0 \\
\Delta x_{14}=\Delta h
\end{gathered}
$$

Substitute the above-mentioned known values into the formula below:

$$
\begin{aligned}
& A_{n c d}^{e x}=A_{n c d 1}^{e x}+A_{n c d 2}^{e x} \\
& =\left(A_{n c d 13}^{e x}+A_{n c d 14(2)}^{e x}+A_{n c d 14(3)}^{e x}\right)+A_{n c d 23}^{e x} \\
& =\left(\frac{1}{2} f_{13} \Delta x_{13}+\frac{1}{2} f_{14(2)} \Delta x_{14}+\frac{1}{2} f_{14(3)} \Delta x_{14}+\frac{1}{2} f_{23} \Delta x_{23}\right. \\
& =\left[\frac{1}{2} f_{13}+\frac{1}{2} f_{14(2)} \quad \frac{\pi}{2} \Delta x_{14}+\frac{1}{2} f_{14(3)} \quad \pi \Delta x_{14}+\frac{1}{2} f_{23}\right. \\
& =-\frac{1}{2} \mu m_{1} g \Delta h \cos \theta
\end{aligned}
$$

Non-conservative external forces do dissipated work to the particle system so that part of mechanical energy transferred to the particle system is converted into the non-mechanical energy in the particle system.

Substitute Equations (49) and (52) into (48) to obtain: 


$$
A_{n c d}=-\frac{1}{2} \mu m_{1} g \Delta h \cos \theta
$$

- Calculate total dissipated work of the system by the non-conservative reacting forces:

From Equation (19), the following is obtained:

$$
A_{n c r d}=A_{n c d}=-\frac{1}{2} \mu m_{1} g \Delta h \cos \theta
$$

- Calculate total implicated work of the system by the non-conservative forces:

Terms with known value:

The implicated displacement of the Particle A's force-applying point by the light rope equals to the displacement of Particle A's force-bearing point:

$$
\Delta x_{3}=\Delta x_{1}=\Delta h .
$$

Using the slope as reference, there is no implicated displacement:

$$
\Delta x_{4}=0 \text {. }
$$

The implicated displacement of the Particle B's force-applying point by the light rope equals to the displacement of Particle B's force-bearing point:

$$
\Delta r_{3}=\Delta x_{2}=\Delta h .
$$

Substitute (61), (62) and (63) into the equation below:

$$
\begin{aligned}
& A_{n c i}^{e x}=\left(A_{n c i 13}^{e x}+A_{n c i 14(2)}^{e x}+A_{n c i 14(3)}^{e x}\right)+A_{n c i 23}^{e x} \\
& =\left(f_{13} \Delta x_{3}+f_{14(2)} \Delta x_{4}+f_{14(3)} \Delta x_{4}+f_{23} \Delta r_{3}\right. \\
& =\left(f_{13} \Delta x_{3} \cos 0+f_{14(2)}+f_{14(3)}+f_{23} \Delta r_{3} \quad \pi\right. \\
& =0
\end{aligned}
$$

- Calculate the increment of kinetic energy of particle system for the whole journey

$$
\begin{aligned}
& \left\{\begin{array}{c}
v_{10}=v_{20}=0 \\
v_{1}=v_{2}
\end{array} \rightarrow\right. \\
& E_{k}-E_{k 0}=\sum_{i=1}^{2}\left(\frac{1}{2} m_{i} v_{i}^{2}\right)-\sum_{i=1}^{2}\left(\frac{1}{2} m_{i} v_{i 0}^{2}\right) \\
& =\left(\frac{1}{2} m_{1} v_{1}^{2}+\frac{1}{2} m_{2} v_{2}^{2}\right)-0 \\
& =\frac{1}{2}\left(m_{1}+m_{2}\right) v_{1}^{2}
\end{aligned}
$$

- Get equations to work out the unknown quantities 
Substitute Equations (53), (59), (60), (64) and (66) into the particle system NTKE equation (44) to obtain:

$$
\left(m_{2}-\frac{1}{2}\left(m_{1}+m_{2}\right) v_{1}^{2} m_{1} \sin \theta\right) g \Delta h-\frac{1}{2} \mu m_{1} g \Delta h \cos \theta-\frac{1}{2} \mu m_{1} g \Delta h \cos \theta+0=\frac{1}{2}\left(m_{1}+m_{2}\right) v_{1}^{2}
$$

The result is:

$$
v_{1}=v_{2}=\sqrt{\frac{\left(m_{2}-m_{1} \sin \theta-\mu m_{1} \cos \theta\right)}{m_{1}+m_{2}} 2 g \Delta h}
$$

Discussion: Only when $m_{2}-m_{1} \sin \theta-\mu m_{1} \cos \theta>0$, the particle system can do the motion in the above-assumed condition.

\section{Conclusion}

The NTKE on particle system is derived from that on particles. NTKE, whether on particles or on particle system, can reveal actual transferring and conversion of mechanical energy and necessarily provide correct guidance to practices. The NTKE shall be flexibly used on the basis of practical conditions and requirements to set and solve equations.

\section{Reference:}

[1] Yang ,Y. (2010). Study on new work-energy theory. New Course Learning, 33, 184-185.

[2] Yang, Y. (2014). Discussion on particle's absolute, relative and implicated motions. Compilation of Scientific Educational Papers, 11, 49-50.

[3] Zhou, Y. B. (1979). Course on Theoretical Mechanics, 19-23. Beijing: Higher Education Press.

[4] Yang, Y. (2002). Discussion on the deductive theories of system work-energy principle. Academic Journal of Shaanxi Normal University, 30, 24-26.

[5] Yang, Y. (2005). Demonstration and dissemination of deduction from system work-energy theory. Journal of Shaanxi Normal University (for Natural Science), 33, 12-15, 292.

[6] Yang, Y. (2009). Acting-force does work, so does the reacting force: Automation of manufacture, 12, 126-128.

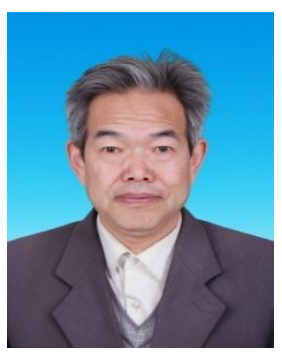

Yang Yong was born in Tongchuan city, Sha'anxi province, China, in November 1959. He is an associate professor in physics and a contract research fellow of both Center for Future Studies, China Society for Future Studies, and of Chinese Academy of Management Science.

He graduated from the Department of Physics with Sha'anxi Normal University in July 1983 for the bachelor degree and from Graduate School of Sha'anxi Normal University majoring in curriculum and teaching theory in 2004 for master degree. He had been teaching physics at the Department of Mathematics and Physics in Tongchuan College of Education and Tongchuan Vocational and Technical College for 34 years.

With research focused on Dynamics of Particles, he had published papers such as "on a derivation from the theory of work-energy of systems and study on new theory of work-energy." He revealed theoretically and discovered "non-conservative force does dissipated work, so does its reacting force and non-conservative force also does non-dissipated work" firstly, and then by further derivation came up with the new theory of work-energy. In this way, he unified the theory of work-energy and practices at a new level and thus developed the classical theory on work-energy into a new course. 